\title{
Multicolor imaging in choroidal osteomas
}

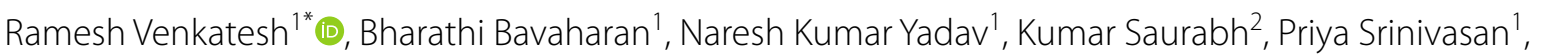 \\ Padmalini Mahendradas ${ }^{1}$, Vishma Prabhu' ${ }^{1}$ and Rupak Roy ${ }^{2}$
}

\begin{abstract}
Background: To describe the multicolour (MC) imaging characteristics associated with choroidal osteomas (CO) and their secondary complications.

Methods: Retrospective descriptive case series of eleven eyes of ten patients with CO. Findings of multicolour imaging were correlated with visual acuity, clinical features, lesion characteristics and findings from other imaging modalities.

Results: Infrared reflectance (IR) images showed calcified CO lesions as hyporeflectance (dark) areas while decalcified lesions were seen as iso reflectance (normal) areas. Overlying RPE atrophy on IR were seen as white areas. MC images showed color variations depending upon the reflectivity of the tumour material tumour and retinal pigment epithelial (RPE) atrophy. Green color was noted in calcified CO tumour while decalcified CO tumour showed no color change. RPE atrophy were seen as bright orange areas. Green and blue reflectance images were not able to pick the choroidal osteoma lesion. Other changes secondary to CO like presence of choroidal neovascular membrane, hemorrhage and/ or fluid in the retinal layers were identified on green and blue reflectance images.
\end{abstract}

Conclusion: $M C$ imaging is a useful tool in our arsenal of existing imaging modalities in the assessment of $\mathrm{CO}$ and its secondary changes. Change in reflectance of the IR and MC images can be used as an indicator to assess the extent of tumour decalcification and its secondary changes and therefore, can aid in prognostication in the same. It has the potential to replace color fundus photography in documentation and follow up of patients with CO.

Keywords: Choroidal osteoma, Multicolour, Imaging, Optical coherence tomography, Fundus autofluoroscence

\section{Background}

Choroidal osteoma (CO) was first described by Gass et al. [1] in 1978 as a rare benign ossifying tumour of the choroid. It is typically unilateral in $80 \%$ of cases and is seen in young healthy females. It arises in late childhood or early adulthood and its most common symptoms are blurred vision, metamorphopsia and presence of a scotoma [2]. The $\mathrm{CO}$ tends to be located in the juxta or peri papillary area and may extend into the macular region. The clinical appearance of the tumour may vary from well-defined, white-cream or yellow-grey to orange lesion which corresponds to the grade of ossification (orange pigmentation is present in areas with more ossification) [3]. Varying degrees of clumping of orange, yellow

\footnotetext{
*Correspondence: vramesh80@yahoo.com

${ }^{1}$ Department of Retina and Vitreous, Narayana Nethralaya, \#121/C, 1st R

Block, Chord Road, Rajaji Nagar, Bengaluru, Karnataka 560010, India

Full list of author information is available at the end of the article
}

and brown pigment can be identified on the tumour surface. Multiple retinal imaging modalities have been used for studying $\mathrm{CO}$ and its secondary ocular findings. Over time, ocular ultrasound (US), fluorescein angiography (FA) and optical coherence tomography (OCT) have been widely used for diagnosis and follow-up of eyes with CO. Enhanced-depth imaging OCT (EDI-OCT) is a recent addition of OCT, that has been able to reveal the presence of bone lamella, tubular lamella with optically empty centre, vascular channels and trabecular bone in patients with $\mathrm{CO}[4,5]$. Multicolor (MC) scanning laser imaging is a recently introduced innovative technology developed for Spectralis SD-OCT (Heidelberg Engineering, Heidelberg, Germany). It has been widely used to describe the findings in various retinal pathologies [6]. MC imaging is a novel, non-invasive retinal imaging modality that simultaneously acquires three reflectance images of the retina using light of 3 different wavelengths 
and then producing a composite image, thereby allowing analysis of changes at various levels within the retina [7]. $\mathrm{MC}$ imaging findings in eyes with $\mathrm{CO}$ have not yet been described.

The aim of the study is to describe the MC imaging features in $\mathrm{CO}$ in conjunction with color fundus photography (CFP), fundus autofluoroscence (FAF) and OCT.

\section{Methods}

In this retrospective study, $\mathrm{CO}$ cases who had undergone multimodal fundus imaging were collected from 2 tertiary retina referral centres (Narayana Nethralaya, Bangalore and Sankara Nethralaya, Kolkata). The diagnosis was based on the presence of a yellow-white to orangered mass deep to the retinal pigment epithelium (RPE) and bone density on ultrasonography. All patients had undergone CFP, FAF, OCT and MC imaging in the study. Institutional review board approval was obtained for this retrospective study.

The clinical details of each patient were collected retrospectively by review of records, including age, gender, laterality and ocular symptoms. Each patient underwent complete ocular examination including best-corrected visual acuity, slit-lamp examination, and indirect ophthalmoscopy. Lesion characteristics recorded for each eye included lesion location within the fundus, extent of calcification or decalcification, and the presence or absence of sub retinal fluid (SRF) or intraretinal fluid (IRF) with/ without choroidal neovascular membrane (CNV).

Color fundus photography was done using either Optos Daytona ultra-wide field color fundus camera (Optos Daytona; Optos PLC, United Kingdom) or Zeiss 450 Fundus camera. Data included foveal involvement by the tumour (foveal, extrafoveal), tumour color (yellow, orange, white) and presence of calcification or decalcification. Calcification was defined as an orange lesion in the fundus located deep underneath the intact RPE and no visible choroidal vessels. Decalcification (complete, partial) was defined as pale areas within the osteoma, with overlying RPE thinning and visibility of underlying choroidal vessels.

All patients had OCT, FAF and MC imaging performed by confocal scanning laser ophthalmoscope using a Heidelberg Spectralis HRA-OCT (Heidelberg Engineering, Dossenheim, Germany). OCT data included tumour surface configuration (flat or depressed), effects of tumour on overlying retina (RPE, photoreceptor and inner retina status), presence of SRF/IRF with/without CNV. Tumour depth was measured using the calliper function through the epicentre of the tumour. The findings of FAF were described as iso, hypo or hyper AF. The patterns described on $M C$ imaging with infrared reflectance (IR), green reflectance (GR) and blue reflectance (BR) were described as dark areas (hypo) or white areas (hyper) or no changes. On MC images, the color of the lesion varied from greenish to yellowish orange to orange depending upon the state of tumour calcification and RPE atrophy. The scanning field was $30^{\circ}$ in most cases. $55^{\circ}$ field images were obtained in eyes with large sized lesions. In addition, FA, ICGA and OCT-A were performed in select patients only when a suspicion of $\mathrm{CNV}$ was present.

We analysed the MC imaging features in $\mathrm{CO}$ and corelated it with that of CFP, FAF and OCT using the HRAOCT unit.

\section{Results}

There were 11 eyes of 10 Asian patients included in the study. The clinical and demographic features are mentioned in Table 1. There were equal number of males and females in the study ( 5 each). The mean age of patients was 30.63 years (range 16-44 years). Right eye and left eye were involved in 5 and 6 eyes respectively. One patient had bilateral $\mathrm{CO}$ while the remaining 9 patients had unilateral CO. Initial symptoms included blurred vision in 7 patients, metamorphopsia in 1 patient and 2 patients were asymptomatic. Visual acuity was between $20 / 20$ and $20 / 200$ in 8 eyes and $<20 / 200$ in 3 eyes. Poor visual acuity (20/200 or worse) was related to foveal photoreceptor layer loss overlying the decalcified tumour in one eye and subfoveal CNV in 2 eyes. On examination, the tumour looked completely calcified in 4 eyes, partly decalcified in 4 eyes and totally decalcified in 3 eyes. Features of CO on CFP, OCT, FAF and MC imaging are described in detail in Table 2. On CFP, the color of the tumour varied from orange $(n=3)$ to yellowish orange $(\mathrm{n}=5)$ to greyish white $(\mathrm{n}=3)$ depending upon the extent of ossification (orange indicating complete ossification, yellowish orange indicating incomplete decalcification and greyish white indicating incomplete decalcification). The tumour involved the fovea in 9 eyes and was extrafoveal in location in 2 eyes only.

The OCT data was recorded by evaluating the scans passing through the macula and over the $\mathrm{CO}$ lesion. The mean central retinal thickness was $259.91 \mu \mathrm{m}$ (range 146-328 $\mu \mathrm{m}$ ). The mean tumour thickness was $625.3 \mu \mathrm{m}$ (range 407-956 $\mu \mathrm{m}$ ). The retinal pigment epithelium (RPE) overlying the tumour was intact in 6 eyes while it was disrupted/irregular in remaining 5 eyes. In addition, $\mathrm{CNV}$ formation was noted in 5 eyes, focal choroidal excavation was seen in 4 eyes while SRF without CNV was seen in 2 eyes. 
Table 1 Demographic and clinical findings in patients with choroidal osteoma

\begin{tabular}{|c|c|c|c|c|c|c|c|c|c|}
\hline \multirow[t]{2}{*}{ Patient } & \multirow[t]{2}{*}{ Involved eye } & \multirow[t]{2}{*}{ Age } & \multirow[t]{2}{*}{ Symptoms } & \multirow[t]{2}{*}{ VA } & \multicolumn{4}{|l|}{ Clinical examination } & \multirow[t]{2}{*}{ Treatment } \\
\hline & & & & & Tumour color & $\begin{array}{l}\text { Foveal } \\
\text { involvement }\end{array}$ & Type & $\begin{array}{l}\text { Associated } \\
\text { ocular } \\
\text { findings }\end{array}$ & \\
\hline 1 & RE & $23 / \mathrm{M}$ & None & $20 / 20$ & Orange & Yes & C & MNF & Observation \\
\hline 2 & LE & $22 / \mathrm{M}$ & BOV & $20 / 400$ & Orange & Yes & C & FCE & Observation \\
\hline 3 & LE & $16 / M$ & $\mathrm{BOV}$ & $20 / 30$ & Yellow & Yes & DC & $\mathrm{Nil}$ & Observation \\
\hline \multirow[t]{2}{*}{4} & RE & $41 / F$ & BOV & $20 / 20$ & Yellowish orange & Yes & $C$ & Nil & Observation \\
\hline & LE & $41 / F$ & BOV & $20 / 400$ & Greyish white & Yes & DC & $\mathrm{FCE}, \mathrm{CNV}$ & IV Anti-VEGF \\
\hline 5 & RE & $40 / M$ & Metamorphosia & $20 / 80$ & Greyish white & Yes & DC & $\mathrm{FCE}, \mathrm{CNV}$ & IV Anti-VEGF \\
\hline 6 & RE & $37 / F$ & BOV & $20 / 60$ & $\begin{array}{l}\text { Yellowish orange with white areas } \\
\text { in the macula }\end{array}$ & Yes & PD & CNV & IV Anti-VEGF \\
\hline 7 & LE & $36 / M$ & None & $20 / 20$ & Orange & No & C & $\mathrm{Nil}$ & Observation \\
\hline 8 & LE & $18 / F$ & BOV & $<20 / 400$ & Yellowish orange with fresh SRH & No & PD & CNV & IV Anti-VEGF \\
\hline 9 & RE & $19 / F$ & BOV & $20 / 60$ & $\begin{array}{l}\text { Greyish-white with yellowish pig- } \\
\text { mentation noted at the macula }\end{array}$ & Yes & PD & FCE & Observation \\
\hline 10 & LE & $44 / F$ & BOV & $20 / 30$ & Yellowish orange & Yes & PD & Scarred CNV & Observation \\
\hline
\end{tabular}

$V A$ visual acuity, $R E$ right eye, $L E$ left eye, $M$ male, $F$ female, $B O V$ blurring of vision, $S R H$ sub retinal hemorrhage, $C$ calcified, $P D$ partly decalcified, $D C$ decalcified, $M N F$ myelinated nerve fibre, FCE focal choroidal excavation, CNV choroidal neovascular membrane, VEGF vascular endothelial growth factor

Fundus autofluorescence findings varied depending on the ophthalmoscopic appearance of each lesion. Heterogeneous lesions exhibited multiple FAF patterns that depended on the characteristics of those corresponding portions of the lesions. Of the 11 eyes with lesions, 5 eyes had areas of iso AF while the remaining 6 eyes had a combination of hyper and hypo AF areas. The findings on FAF depended on the tumour characteristics like the extent of calcification and also on associated retinal findings like presence of CNV, hemorrhage, SRF/IRF and RPE atrophy.

$\mathrm{MC}$ imaging was performed in all eyes using the cSLO Heidelberg Spectralis HRA-OCT (Heidelberg Engineering, Dossenheim, Germany) with a scanning field of $30^{\circ}$ and sometimes $55^{\circ}$. IR images were the most efficient in identifying CO. The IR images identified ossified portions of the tumour as dark (hypo reflectance) areas while deossified portions showed iso-reflectance (no change) areas. White (hyper reflectance) areas were seen in RPE atrophy. The GR and BR images showed either no change or white areas depending upon the presence of hemorrhage and/or fluid in the retinal layers. On MC images, the color of the lesion varied from being green to no change to bright orange depending upon the tumour content and RPE atrophy. Green color was noted in calcified tumour while decalcified tumour showed no change on multicolour images. Orange areas were noted in eyes with areas of decalcified tumour with overlying RPE atrophy (Figs. 1, 2, 3, 4).

Seven eyes with SRF/IRF and clinical suspicion of choroidal CNV were imaged further with FA and ICGA and OCT- angiography in some cases. CNV was identified in 5 eyes and treatment were administered with anti-VEGF therapy in 4 eyes. One eye had scarred CNV; hence, it was observed.

\section{Discussion}

Multi color scanning laser imaging is a recently introduced non-invasive innovative technology developed for Spectralis SD-OCT (Heidelberg Engineering, Heidelberg, Germany). In scanning laser ophthalmoscopy, a single point of laser light of a specified wavelength is scanned across the retina in a series of parallel lines and enabling an alternative method of capturing fundus images [8,9]. It uses three laser colours, blue $(488 \mathrm{~nm})$, green $(515 \mathrm{~nm})$ and infrared $(820 \mathrm{~nm})$ that penetrate the tissue to different depths and simultaneously captures the reflectance strengths from different retinal structures and represents the information as en-face images. The infrared reflectance (IR) image visualizes structures at the level of the outer retina, RPE and choroid. Thus, the higher wavelength infrared light is useful in depicting and characterizing the $\mathrm{CO}$ lesion. The GR image allows imaging of retinal blood vessels, haemorrhages and exudates. The 


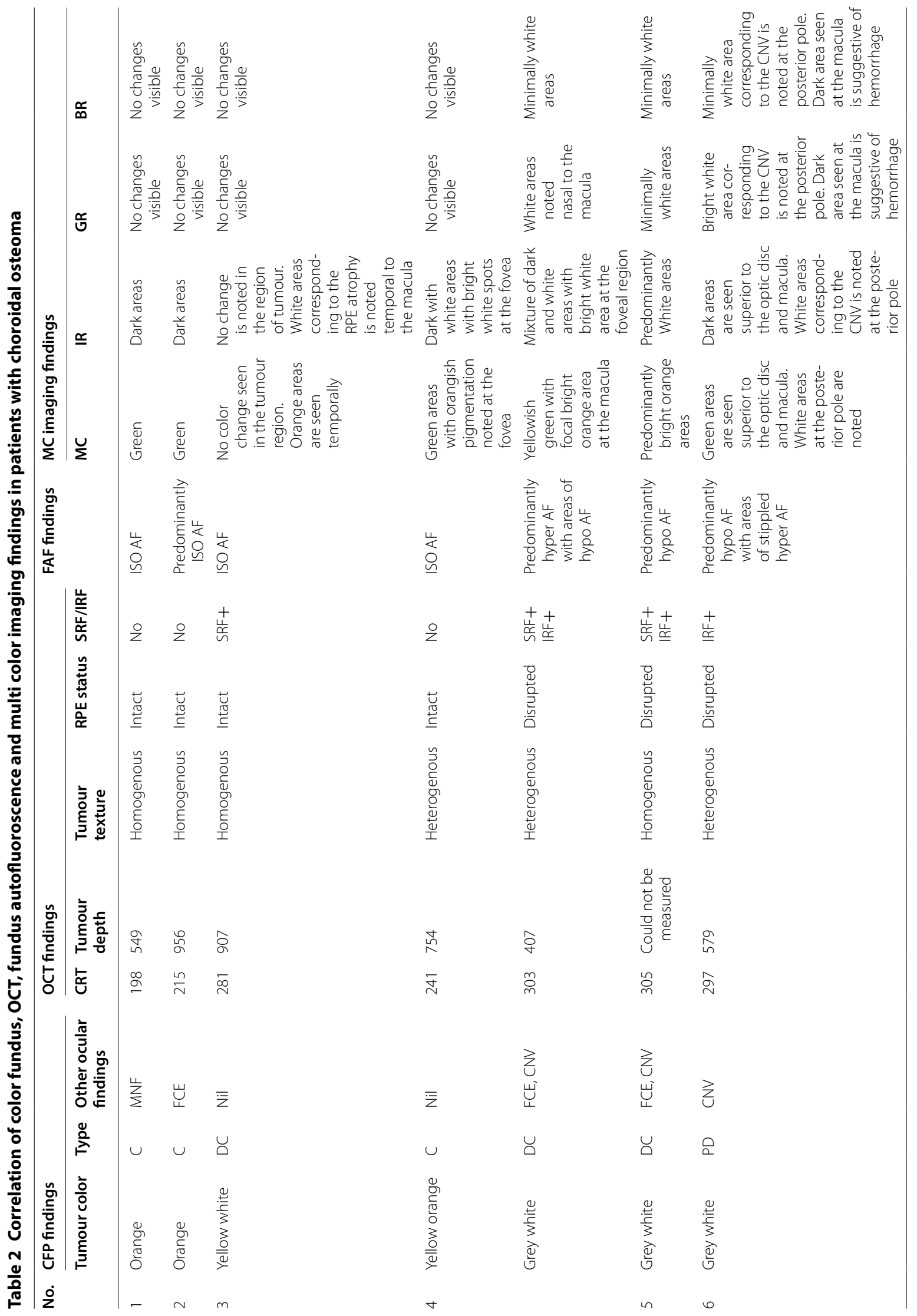




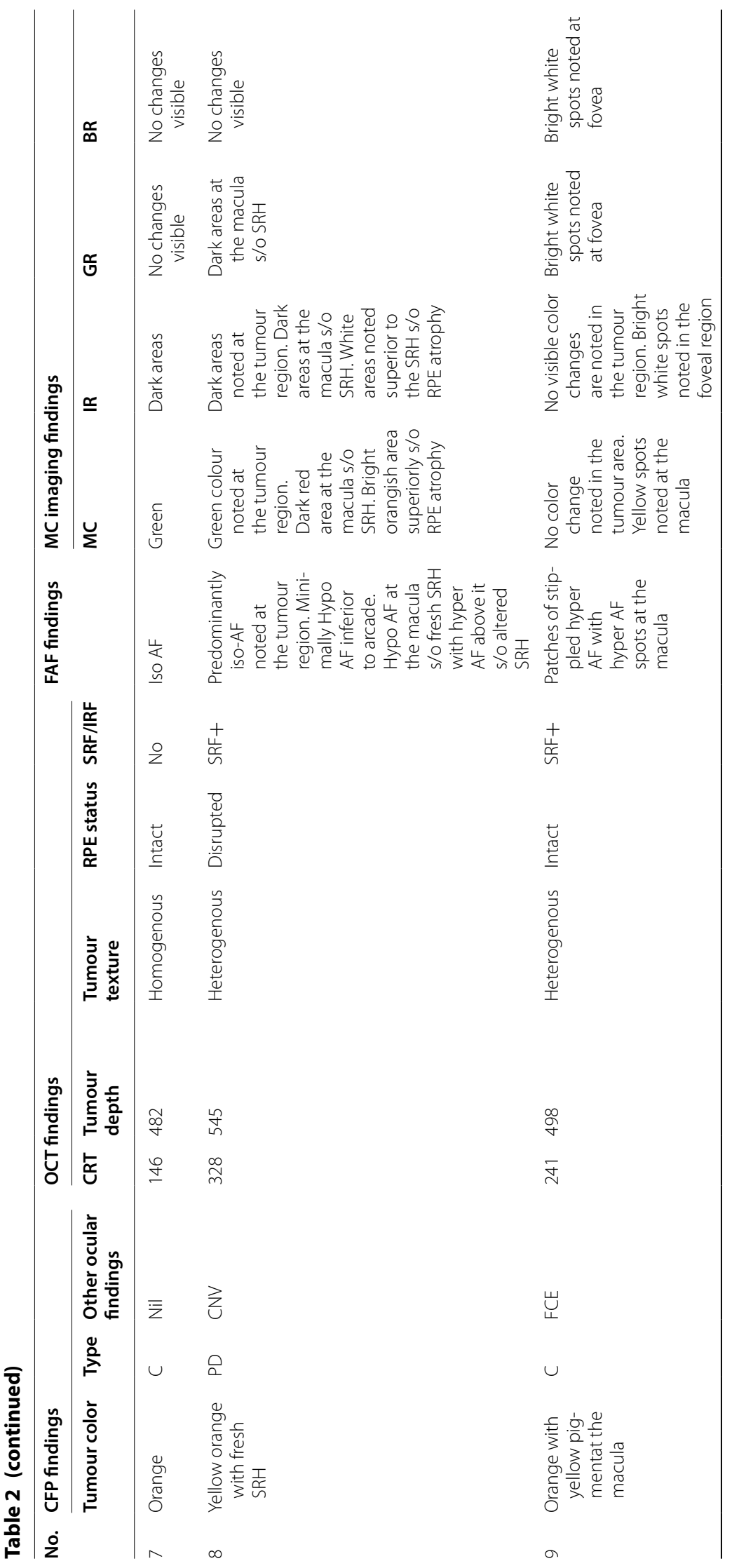




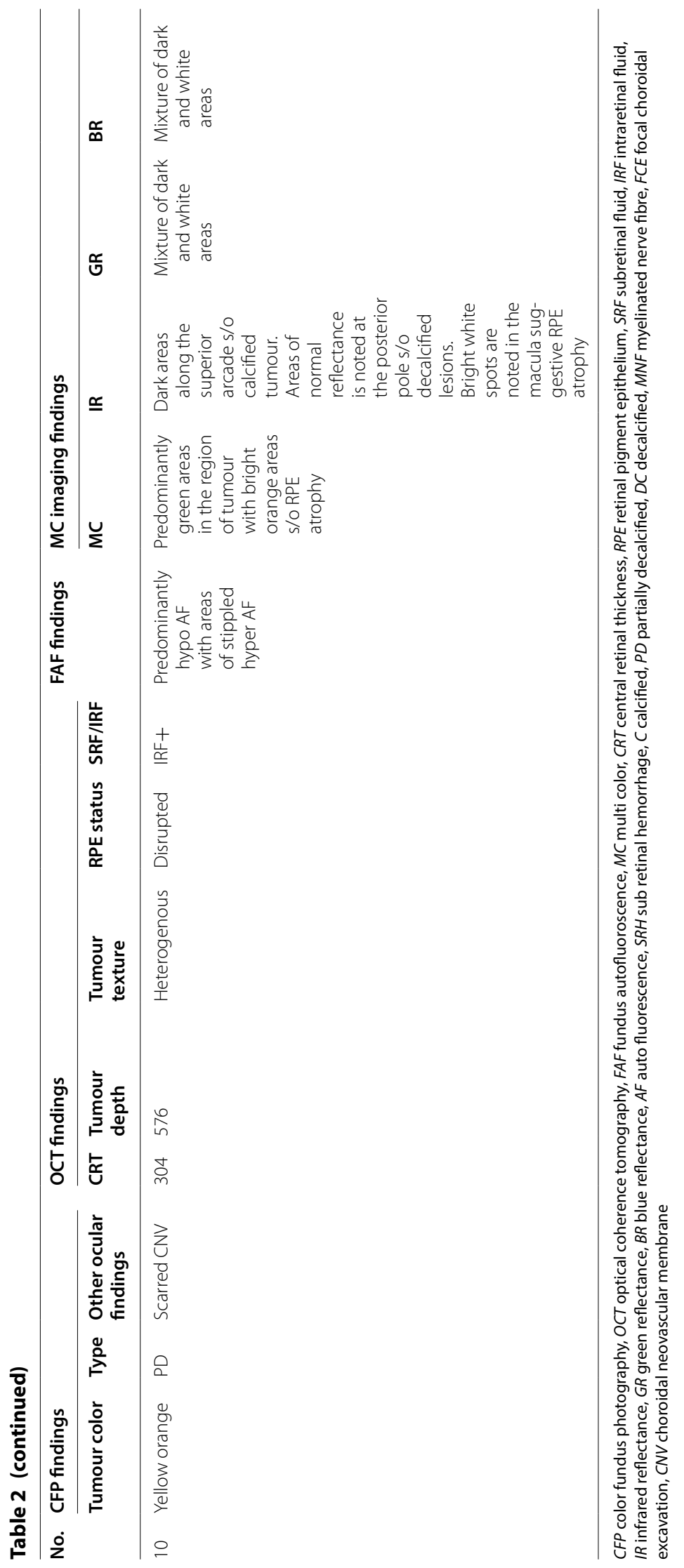




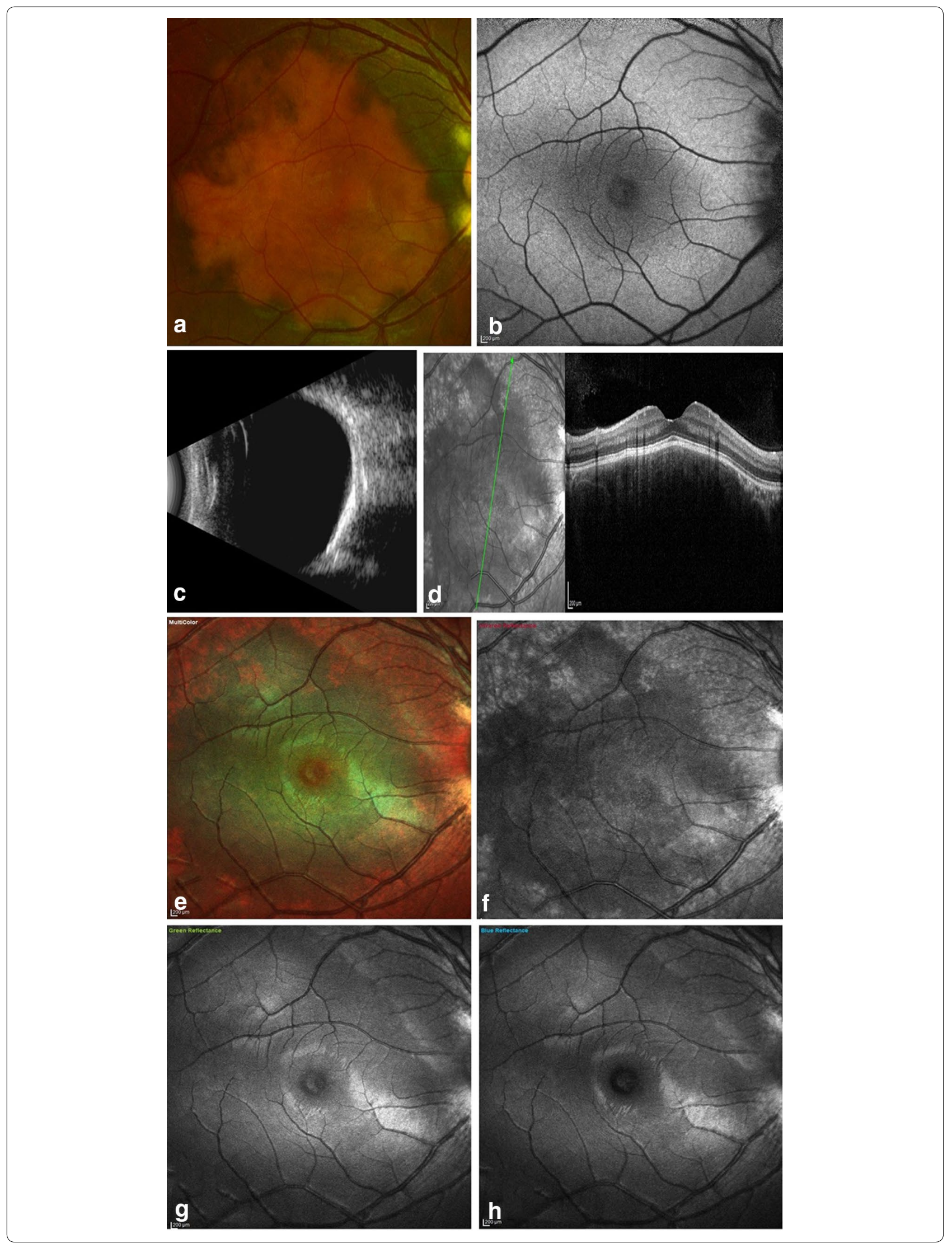


(See figure on previous page.)

Fig. 1 Case 1-Multimodal imaging with color fundus (CF), fundus autofluoroscence (FAF), optical coherence tomography (OCT), ultrasonography (USG) and multicolour (MC) imaging in a patient with RE calcified choroidal osteoma. a CF showing RE orange coloured lesion underneath the retinal pigment epithelium (RPE) at the posterior pole. $\mathbf{b}$ FAF showing iso autofluoroscence in the region of interest. $\mathbf{c}$ High bone density noted on USG b-scan. $\mathbf{d}$ Radial OCT scan passing thru'the lesion shows retinal elevation with overlying normal retinal contour. e MC image showing the green colouration at the posterior pole. $\mathbf{f}$ Infrared reflectance showing dark areas corresponding to the tumour lesion. $\mathbf{g}, \mathbf{h}$ Green and blue reflectance images do not show any changes due to $\mathrm{CO}$ at the posterior pole

BR particularly provides details of the inner retina and the vitreoretinal interface such as epiretinal membranes, retinal nerve fibre layer (RNFL) thinning and macular pigment changes. Thus, secondary retinal findings like presence of intraretinal/subretinal hemorrhage, fluid or pigments are picked up with GR and BR images. The information from these 3 images are integrated to form a composite MC image [7]. In this study, we describe the features of $\mathrm{CO}$ on $\mathrm{MC}$ imaging and corelate them with that of CFP, FAF and OCT.

$\mathrm{CO}$ is a rare benign ossifying tumour of the choroid characterised by the presence of mature, spongy cancellous bone. On histopathology, dense bony trabeculae with marrow spaces traversed by pathognomonic dilated thin-walled blood vessels, termed spider or feeder vessels are seen. These vessels connect the choriocapillaris to the underlying larger choroidal vessels [1]. Choroidal imaging using the EDI-OCT has revealed the presence of bone lamella, tubular lamella with optically empty centre, vascular channels and trabecular bone in patients with $\mathrm{CO}[4,5]$. Because of its deeper location, $\mathrm{CO}$ are best picked up on IR images. The IR image shows hypo reflectance (dark) areas in an ossified tumour. This is probably because the $820 \mathrm{~nm}$ infrared light gets absorbed by the spongy bony trabeculae present in CO. As a result, very less quantity of light gets reflected back and hence, the lesion appears as a dark area. In decalcifying tumours, there is absence of spongy, bony trabeculae resulting in an iso-reflectance pattern of the tumour. Areas of RPE atrophy are identified as hyper reflectance (white) areas on IR image. In the BR and GR images, the CO lesion is not visible. This is due to the inability of the shorter blue and green wavelength light to reach the $\mathrm{CO}$ lesion. However, in the presence of secondary changes like pigmentary deposition and presence of intraretinal fluid and hemorrhage due to $\mathrm{CNV}$, areas of abnormal reflectance are identified on BR and GR images.

Fundus autofluoroscence images does not show the osteoma lesion when the overlying RPE is intact. However, in areas of atrophic RPE, hypo AF (dark) areas are seen.

The $\mathrm{MC}$ image in $\mathrm{CO}$ shows color variations due to the variable reflectance produced by the tumour. The green colour on MC images are seen in eyes with calcified tumour while decalcified choroidal osteoma shows no colour change. Areas of RPE atrophy are seen as brightorange areas. Pigment clumping over the tumour surface is usually difficult to identify ophthalmoscopically against the orange intrinsic tumour color. On MC images, pigment clumps are identified as yellow or orange spots while on IR images, they are seen as bright white spots.

Poor visual acuity in eyes with $\mathrm{CO}$ is usually seen when the decalcification with overlying atrophy of RPE and photoreceptors is located underneath the fovea. Thus, presence of bright orange areas on $\mathrm{MC}$ image and white areas on IR image at the sub foveal region can predict

\footnotetext{
(See figure on next page.)

Fig. 2 Case 3-Multimodal imaging in a patient with predominantly decalcified choroidal osteoma in the LE. a CF showing yellowish choroidal osteoma lesion at the peripapillary region with retinal pigment epithelial alterations noted temporal to the macula. $\mathbf{b}$ Ocular ultrasound showed hyperechoic area with shadowing confirming CO. c Horizontal line OCT scan passing through the fovea shows subtle elevation by the tumour temporal to the $\mathrm{ONH}$ and presence of sub foveal SRF. $\mathbf{d}$ MC image shows minimal orangish discoloration in the peripapillary area and temporal to the macula suggestive of overlying RPE atrophy. The decalcified tumour is not seen on MC image. e Infrared reflectance shows white areas corresponding to the orange areas in MC image suggestive of atrophied RPE. $\mathbf{f}, \mathbf{g}$ Blue and green reflectance images do not show the osteoma lesion
} 

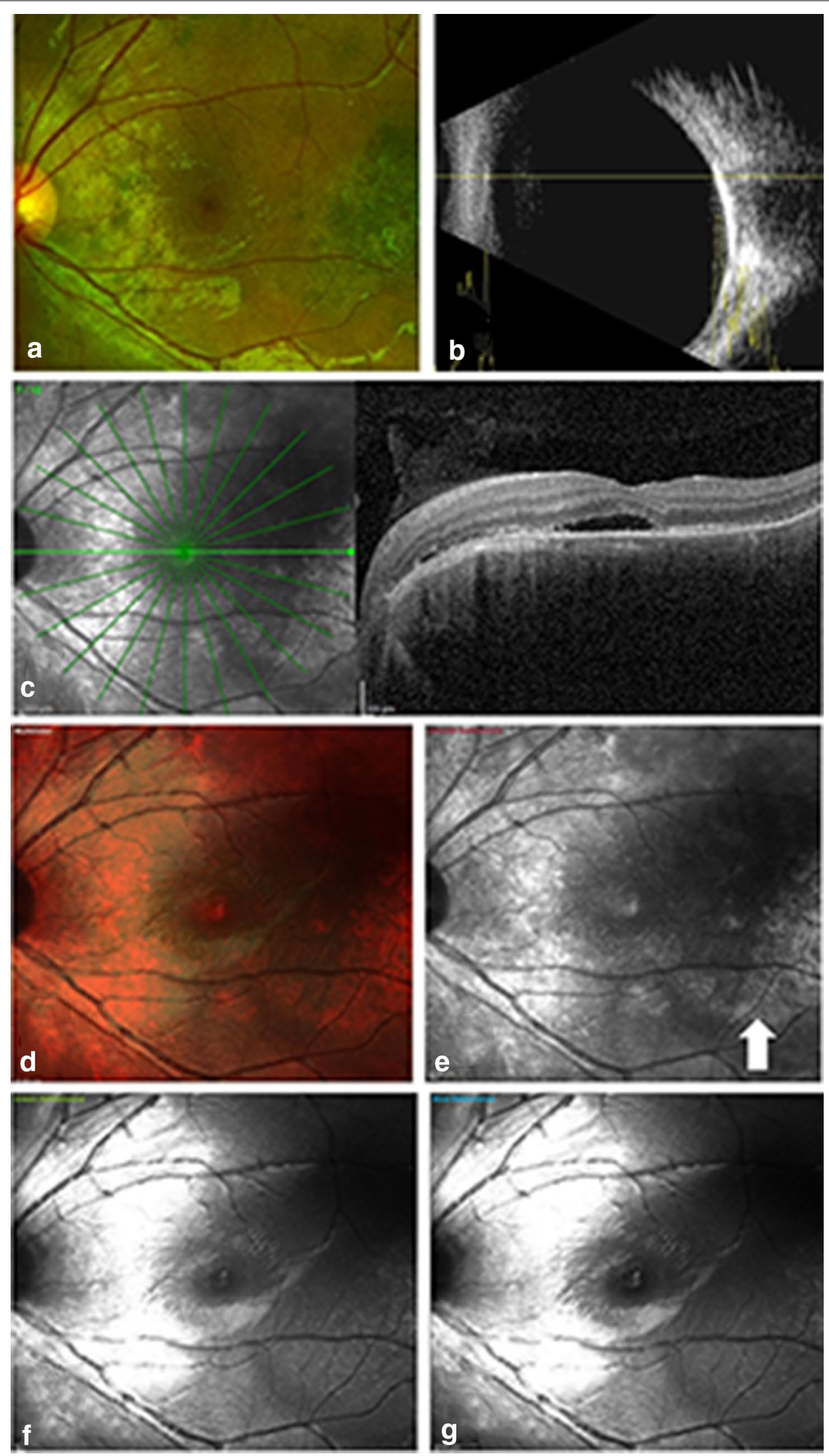


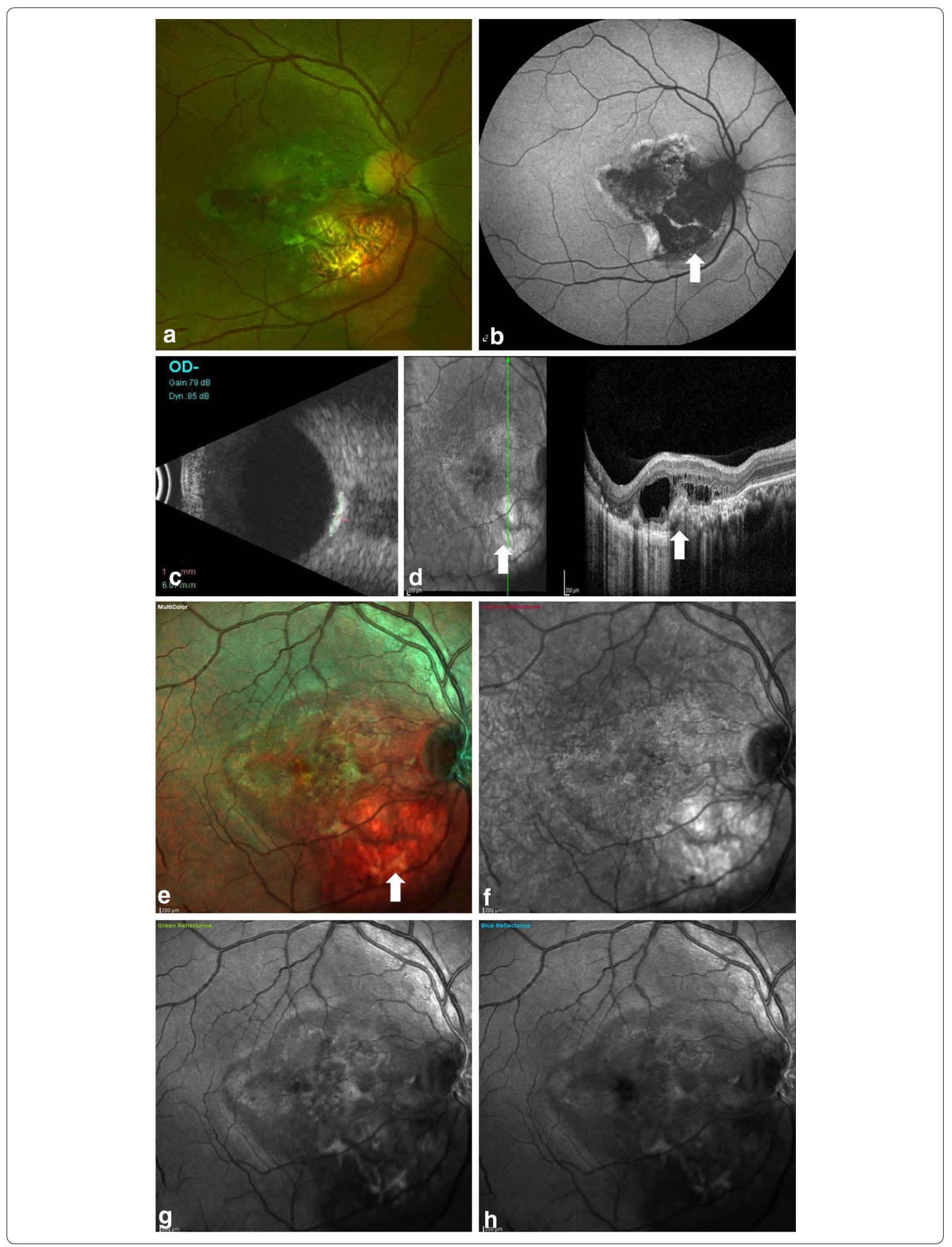




\section{(See figure on previous page.)}

Fig. 3 Multimodal imaging in case 5 with total decalcified choroidal osteoma. a CF showing a greyish white area inferotemporal to the optic nerve head (ONH) with RPE atrophy and prominent visible underlying choroidal vessels. b FAF showing areas of hypo AF suggestive of total RPE atrophy. c High bone density noted on USG b-scan. d Radial OCT scan passing vertically through the lesion shows disrupted RPE inferotemporal to the $\mathrm{ONH}$ with intraretinal fluid. e $\mathrm{MC}$ image shows orange discoloration at the posterior pole with bright orange area noted inferotemporal to the ONH suggestive of RPE atrophy overlying the choroidal osteoma. $\mathbf{f}$ Infrared reflectance shows white areas at the posterior pole and bright white area corresponding to the bright orange area noted on MC image suggestive of RPE atrophy. $\mathbf{g}$, $\mathbf{h}$ Minimally white areas are noted in both green and blue reflectance images

poor visual acuity. Hence, $\mathrm{MC}$ imaging can be considered as a useful tool in disease prognostication.

MC imaging has some advantages over CFP [10]. It is: (1) less photophobic to the patient; (2) can be used in undilated pupil; (3) $55^{\circ}$ images gives a larger view of the periphery (4) can be combined with SD-OCT in a single device to allow simultaneous fundus and cross-sectional imaging and (5) provides images with high contrast and enables imaging through hazy media like cataract. MC imaging also seems to be superior to FAF in identifying the tumour and secondary changes produced by it. In comparison to FA and ICGA, MC imaging is noninvasive, less time consuming, cheaper, and has no side effects due to dye injection.

Our study has the advantage of having sufficient number of eyes with $\mathrm{MC}$ images in a rare disease like CO. We also had the multicolour image findings of the disease in its different stages; thus, helping us to understand better the role of $\mathrm{MC}$ imaging in $\mathrm{CO}$. Our study had several limitations as well. Absent follow-up images meant inability to describe MC imaging changes over time. All ancillary imaging studies were not used in each patient, and raster scans on SD-OCT may have missed pertinent portions of the lesions. Larger series with longer follow-ups and comparison with other imaging modalities will elucidate greater utility of MC imaging for choroidal osteomas. Present study illustrates multicolour imaging features of CO lesion, which is hitherto not described in English language published literature.

\section{Conclusion}

MC imaging is a useful tool in our arsenal of existing imaging modalities in the assessment of $\mathrm{CO}$ and its secondary changes. Changes in IR reflectance and $\mathrm{MC}$ images can be used as an indicator to assess the extent

\footnotetext{
(See figure on next page.)

Fig. 4 Multimodal imaging of a partly decalcified choroidal osteoma in the RE of case 6. a CF image of the RE showing CO lesion at the posterior pole with presence of CNV and subretinal hemorrhage. b High bone density noted on USG b-scan. c Horizontal radial OCT scan passing thru' the macula demonstrating the presence of disrupted RPE with CNV and intraretinal fluid. $\mathbf{d}$ MC image shows green areas superior and temporal to the ONH suggestive of calcified CO. e Infrared reflectance showing dark areas corresponding to the calcifies areas of CO (blue arrows). Area of hyperreflectance is noted at the macula suggestive of CNV (white arrow). $\mathbf{g}, \mathbf{h}$ Green and blue reflectance images shows bright white area corresponding to the CNV at the posterior pole while dark area is noted at the edge of CNV suggestive of hemorrhage (red arrow)
} 

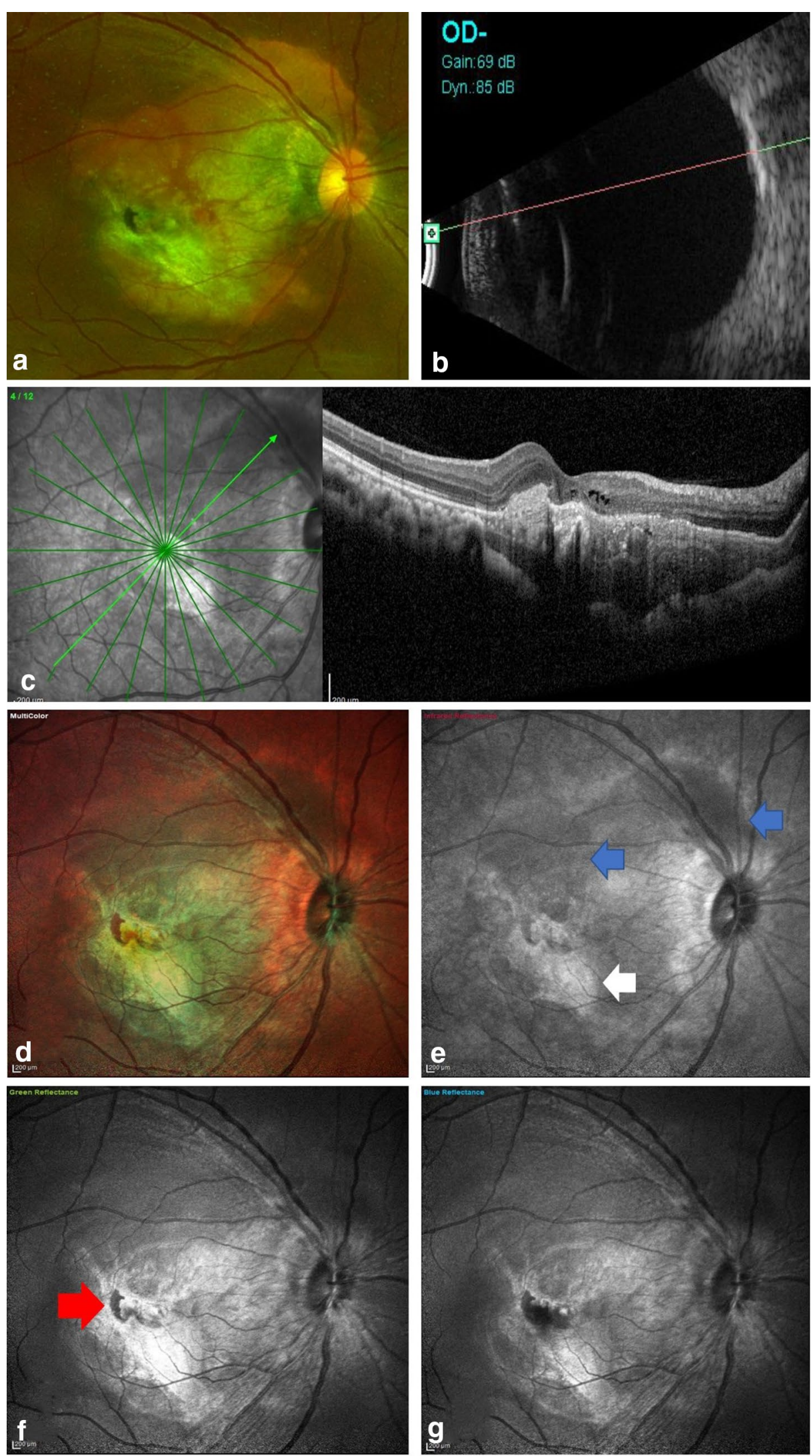
of tumour decalcification and its secondary changes and therefore, can aid in prognostication in the same. It has the potential to replace CFP in documentation and follow up of patients with CO.

\section{Abbreviations}

CO: choroidal osteoma; US: ultrasound; FA: fluorescein angiography; OCT: optical coherence tomography; OCTA: optical coherence tomography angiography; EDI: enhanced depth imaging; MC: multicolour; CFP: colour fundus photography; FAF: fundus autofluoroscence; RPE: retinal pigment epithelium; SRF: subretinal fluid; IRF: intra retinal fluid; CNV: choroidal neovascular membrane; IR: infrared reflectance; GR: green reflectance; BR: blue reflectance; VEGF: vascular endothelial growth factor; $\mathrm{SRH}$ : subretinal hemorrhage.

\section{Authors' contributions}

$\mathrm{RV}$ — conceptualising the study, data acquisition, analysing the data, interpreting the findings, writing \& reviewing the manuscript. BHA-Data acquisition and analysing the data. NKY — reviewing the manuscript. KS, VP, PS \& MPAData acquisition. RKR — data acquisition, analysing the data, interpreting the findings, reviewing the manuscript. All authors read and approved the final manuscript.

\section{Author details}

${ }^{1}$ Department of Retina and Vitreous, Narayana Nethralaya, \#121/C, 1st R Block, Chord Road, Rajaji Nagar, Bengaluru, Karnataka 560010, India. ${ }^{2}$ Department of Retina and Vitreous, Aditya Birla Sankara Nethralaya, No. 147, Mukundapur EM By Pass, Near Purva Jadavpur Thana, Kolkata, West Bengal 700099, India.

\section{Acknowledgements}

None.

\section{Competing interests}

The authors declare that they have no competing interests.

\section{Availability of data and materials}

The datasets used and/or analysed during the current study are available from the corresponding author on reasonable request.

\section{Consent for publication}

The authors certify that they have obtained all appropriate patient consent forms. In the form, the patient has given his consent for his/her images and other clinical information to be reported in the journal. The patients understand that their names and initials will not be published and due efforts will be made to conceal their identity, but anonymity cannot be guaranteed.
Ethics approval and consent to participate

Not applicable.

Funding

None.

\section{Publisher's Note}

Springer Nature remains neutral with regard to jurisdictional claims in published maps and institutional affiliations.

Received: 5 October 2018 Accepted: 10 December 2018

Published online: 13 December 2018

\section{References}

1. Gass JD, Guerry RK, Jack RL, Harris G. Choroidal osteoma. Arch Ophthalmol. 1978;96:428-35.

2. Shields CL, Shields JA, Augsburger JJ. Choroidal osteoma. Surv Ophthalmol. 1988:33:17-27.

3. Shields CL, Sun H, Demirci H, Shields JA. Factors predictive of tumour growth, tumour decalcification, choroidal neovascularization, and visual outcome in 74 eyes with choroidal osteoma. Arch Ophthalmol. 2005;123(12):1658-66.

4. Erol MK, Coban DT, Ceran BB, Bulut M. Enhanced depth imaging optical coherence tomography and fundus autofluorescence findings in bilateral choroidal osteoma: a case report. Arq Bras Oftalmol. 2013;76(3):189-91.

5. Shields CL, Arepalli S, Atalay HT, Ferenczy SR, Fulco E, Shields JA. Choroidal osteoma shows bone lamella and vascular channels on enhanced depth imaging optical coherence tomography in 15 eyes. Retina. 2015:35(4):750-7.

6. Feng HL, Sharma S, Stinnett S, Asrani S, Mruthyunjaya P. Identification of posterior segment pathology with en face retinal imaging using mc confocal scanning laser ophthalmoscopy. Retina. 2018. https://doi. org/10.1097/iae.0000000000002111 (Epub ahead of print).

7. Tan AC, Fleckenstein M, Schmitz-Valckenberg S, Holz FG. Clinical application of MC imaging technology. Ophthalmologica. 2016;236:8-18.

8. Keane PA, Sadda SR. Retinal imaging in the twenty-first century: state of the art and future directions. Ophthalmology. 2014;121:2489-500.

9. Manivannan A, Van der Hoek J, Vieira P, Farrow A, Olson J, Sharp PF, Forrester JV. Clinical investigation of a true color scanning laser ophthalmoscope. Arch Ophthalmol. 2001;119:819-24.

10. Muftuoglu IK, Gaber R, Bartsch DU, Meshi A, Goldbaum M, Freeman WR. Comparison of conventional color fundus photography and MC imaging in choroidal or retinal lesions. Graefes Arch Clin Exp Ophthalmol. 2018:256(4):643-9.
Ready to submit your research? Choose BMC and benefit from:

- fast, convenient online submission

- thorough peer review by experienced researchers in your field

- rapid publication on acceptance

- support for research data, including large and complex data types

- gold Open Access which fosters wider collaboration and increased citations

- maximum visibility for your research: over $100 \mathrm{M}$ website views per year

At $\mathrm{BMC}$, research is always in progress.

Learn more biomedcentral.com/submissions 